\title{
Multi-Body Simulation and Multi-Objective Optimization Applied to Vehicle Dynamics
}

\author{
Fabiano Maggio ${ }^{1, a}$ \\ ${ }^{1}$ EnginSoft S.p.A., Via Giambellino 7, 35129, Padova, Italy
}

Received 13 April 2009, Accepted 14 August 2009

\begin{abstract}
This paper describes a demo application, where modeFRONTIER, a commercial software for design optimization, is coupled with a parametric 2D Multi-Body model of a touring motorcycle. The optimizer is asked to identify the characteristics of the front suspension (telelever scheme) that deliver more safety, more stability, and more riding comfort for a braking maneuver in straight running. All objectives are described through 4 scalar indexes, extracted from the braking kinematics.

As expected, results highlight that trade-off relationships connect the objectives. Only 13, among the 1900 tested configurations, are able to simultaneously improve the 4 indexes. Moreover, no design in this selection assures a decisive improvement of the stability. In order to accomplish this priority requirement, the best design is chosen accepting a slight reduction of stability.
\end{abstract}

Key words: Motorcycle Optimization, Braking Maneuver, Telelever, Multi-Objective Optimization, Design Optimization

\section{Introduction}

Dynamical performances of two wheeled vehicles largely depend on the response of each component. Among all components, tires and suspensions are the most important sub-systems, because their role is to modulate any force going from the road to the chassis $[1,2,3,4]$. Therefore, a deep knowledge of such parts can be the turning key to develop better and safer motorcycles.

Multi-Body Simulation is the most suitable approach to perform vehicle dynamics investigation $[5,6,7]$. By creating parametric models, different configurations can be quickly evaluated, even at the earliest stage of the entire development process. From a business perspective, this means that wrong gateways can be dropped long before they lead to costly and useless real prototypes.

These benefits can be further increased by coupling the Multi-Body Simulation software with modeFRONTIER, which automatically plans and drives sets of simulations in order to perform complex tasks such as Design of Experiment, Parameter Sensitivity Analysis, and Multi-Objective Optimization.

This paper describes a demo application, where modeFRONTIER is asked to identify the parameters of a motorcycle front suspension to deliver more safety, more stability, and more riding comfort for a braking maneuver in straight running. Although the research is targeted to a very specific case, the proposed methodology can be straightforwardly extended to any user-defined running condition. Potentially, it is possible to build a single modeFRONTIER project to optimize different vehicle parts for various maneuvers.

As expected, results highlight that trade-off relationships connect the objectives, hence full optimization is almost impossible. Only 13, among the 1900 tested configurations, are able to simultaneously improve the 4 scalar indexes that have been previously defined to measure the objectives.
Moreover, no design in this selection promises a decisive improvement of the stability. In order to meet these primary requirements, the best design is chosen accepting a slight reduction of stability. The decision task has been totally supported by the Multi Criteria Decision Making tool implemented in modeFRONTIER.

\section{The 2D Motorcycle Model}

The Motorcycle is a complex multi-body system and special efforts are necessary to reliably simulate the overall dynamics. The broad range of literature available on this topic covers all simulation fields, such as time domain behavior [6, 7], optimal maneuver [8, 9], steady trim [10], frequency response [11], modal properties [5, 14, 15], tire dynamics [12], and so on. Since this research aims at highlighting the benefits of implementing an optimizer into the simulation chain, a detailed and validated model is not available. Model features have been implemented following state-of-art guidelines [13] to ensure sufficient result reliability.

As a simple test case, the vehicle behavior at braking during straight line motion is considered. In general, lateral dynamics plays an important role and a detailed 3D model would be recommended to perform thorough investigation. However, a 2D model includes enough features to study the influence of the front suspension on the braking distance, keeping the simulation complexity at reasonable level. Indirect considerations on stability and vehicle comfort can be made in any event by examining the variation of some inplane quantities $[4,14]$.

The motorcycle model used in this research consists of 7 rigid bodies, such as chassis (which includes the rider), rear wheel, swinging arm, front wheel and three bodies for the front suspension with telelever scheme. Bodies are connected using proper joints to obtain the exact number of inplane degrees of freedom. 


\subsection{Front Suspension}

A special description is required for the fully parametric front suspension geometry (Fig.1).

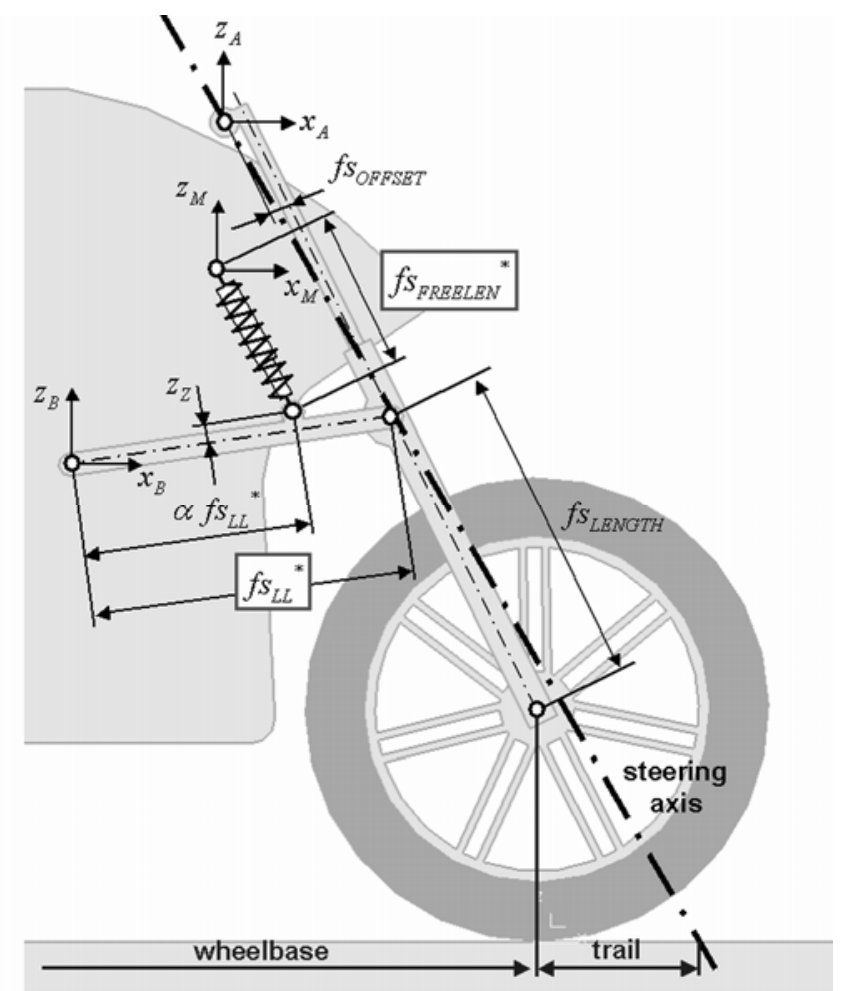

Fig. 1. Front Suspension layout and geometrical parameters (inputs for the optimization problem)

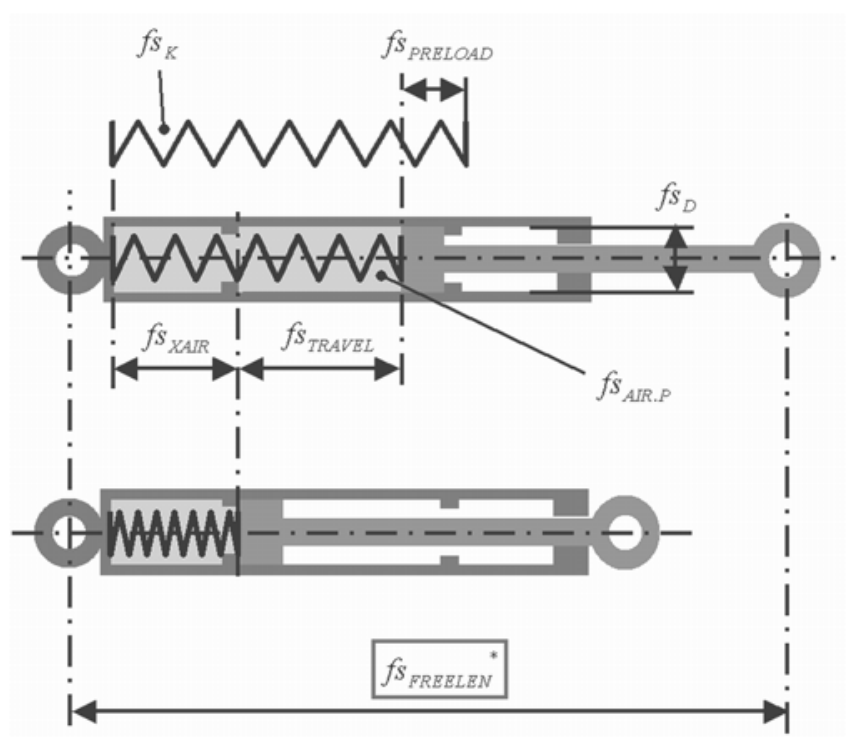

Fig. 2 Parameters for the Spring-Damper assembly (further inputs for the optimization problem)

Parameters allow to change the position of "A", "B", and "M" pins with respect to the chassis. Also, front fork offset, lower fork length, and the main link characteristics are adjustable. Suspension geometry directly affects important vehicle dimensions such as wheelbase, mechanical trail, and center of gravity height. Wheelbase and center of mass po- sition play a fundamental role in determining the vehicle braking response. Since this research only aims at studying the suspension effects on the braking response, some tricks are used to make the above quantities almost independent from the general parameter assignment.

The spring-damper assembly (Fig. 2) is also included in the parameterization. The overall force is obtained by combining the action of a linear spring and a pressurized air piston. The working travel is governed by two high stiff end-stroke pads. Initial preload is obtained by assigning a non-zero air pressure and by imposing a partial compression of the spring. The linear damper has different coefficients for the elongation and compression phases. The free length of the assembly is calculated in order to keep the center of mass height constant when performing the static assembly.

\subsection{Tire}

Tires are modeled to accurately describe the generation of the longitudinal force. In accordance with Pacejka's formula [16], the longitudinal force $S$ is a non-linear function of the longitudinal slip and a linear function of the vertical load. The $D_{x}$ coefficient controls the maximum force that the tire can generate (adherence factor), whereas the others govern the shape of the force function. Maximum occurs for low slip ratio, whereas high slip leads to tire saturation and smaller forces. The tire model implemented here does not take into account any delay between slip and force raising.

Effective rolling radius:

$$
r_{e}=r-\delta
$$

Longitudinal slip:

$$
\kappa=\left(V-\omega r_{e}\right) / V
$$

Vertical load:

$$
N=k_{r} \delta_{r}+c_{r} \dot{\delta}_{r}
$$

Longitudinal force:

$$
\begin{gathered}
\psi=C_{x} \arctan \left\{B_{x} \kappa-E_{x}\left[B_{x} \kappa-\arctan \left(B_{x} \kappa\right)\right]\right\} \\
S=N D_{x} \sin (\psi)
\end{gathered}
$$

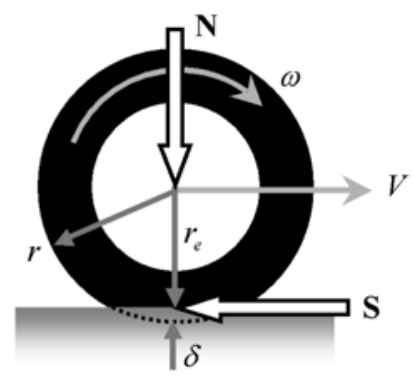

Fig. 3. Tire model 
The simulation reproduces a typical emergency situation where the front brake is activated to stop the motorcycle from straight running at $130 \mathrm{~km} / \mathrm{h}$. The front brake simply reduces the angular speed of the front wheel, increasing the longitudinal slip that modulates the longitudinal braking force. During the entire maneuver, the vertical load is transferred from rear to front tire, proportionally to the deceleration [4]. This means that the front tire becomes capable of generating a greater braking force.

Braking dynamics are governed by two practical limits. In case of good adherence, the front braking force can become high enough to cause the lift of the rear wheel (stoppie condition). On the contrary, with small values of the $D_{x}$ coefficient, the front tire reaches its saturation point and the front wheel locks.

The planned optimization makes sense if a control system (Fig. 4) instantaneously calculates the braking torque that leads to the maximum braking force, with no rear wheel lift. This will fully exploit the motorcycle's braking capabilities. If any suspension update (design generation) affects these capabilities, the simulation will be able to capture such effect.

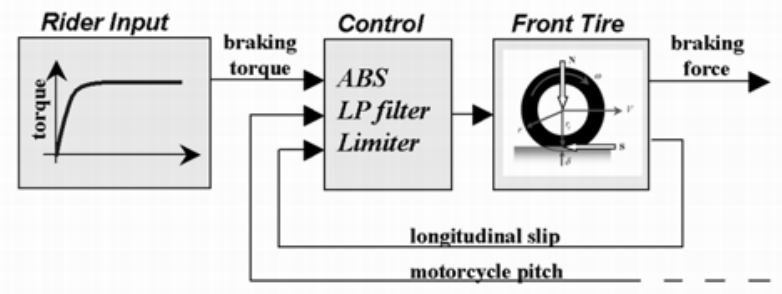

Fig. 4. Braking control

\section{Optimization Problem and Objectives}

The suspension characteristics influence both the way the braking force is generated (transient phase) and its value in steady condition (constant deceleration phase). It is expected that by changing the suspension parameters, it will be possible to obtain a higher force at a shorter interval, and hence a reduction of the total stopping distance. This is the most important objective for the optimization problem.

During the braking maneuver, both the wheelbase and the trail tend to get shorter, usually causing a reduction of the lateral vehicle stability $[13,14]$. These phenomena can be limited by choosing an opportune suspension layout. Therefore, two objectives corresponding to trail and wheelbase shortenings are defined.

Table 1. Objectives and their priority

\begin{tabular}{ccccc}
\hline Quantity & Field & Pr & Baseline & Objective \\
\hline trail variation & stability & 2 & $-7.4 \mathrm{~mm}$ & increase \\
wheelbase variation & stability & 3 & $-5.1 \mathrm{~mm}$ & increase \\
stopping distance & safety & 1 & $81.7 \mathrm{~m}$ & decrease \\
pitch angle variation & comfort & 4 & $3.7 \mathrm{deg}$ & decrease \\
\hline
\end{tabular}

A further consequence of any braking action is the pitching forward of the motorcycle. This is not necessarily a problem, but excessive pitching might disturb the rider. For this reason, we ask the optimizer to identify designs that also limit the pitching.

All of the optimization objectives are listed in Table 1, which includes also the performances given by the baseline motorcycle (i.e. initial design).

\section{Software Implementation}

The parametric motorcycle model has been developed using commercial multi-body software. Geometrical properties for the initial design have been indirectly measured from digital pictures, whereas inertial properties have been estimated. Control routines have been written in FORTRAN code and then linked to the main model. VISUAL BASIC modules have also been written to automatically export time domain results at the end of each simulation.

The workflow is fully defined in the modeFRONTIER environment, which controls any task and, most importantly, drives the optimization using its internal algorithms. The optimization philosophy is to explore the design domain (14 dimensional space) looking for the configurations that improve the system behavior in accordance with the 4 objectives. First, a DOE population with 112 designs is generated to map how the 4 objectives depend on the 14 input variables. Then, the Multi-Objective Genetic Algorithm of modeFRONTIER generates populations of new designs, using the information gained from previous runs.

To test each configuration and extract the corresponding outputs, modeFRONTIER executes internal operations and launches external applications. The sequence of the main tasks is given in Table 2.

Table 2. Process integration tasks

\begin{tabular}{cc}
\hline Action & Linked resource \\
\hline $\begin{array}{c}\text { generation of } \\
\text { input variable set }\end{array}$ & $\begin{array}{c}\text { modeFRONTIER } \\
\text { DOE or MOGA algorithms } \\
\text { assembly of } \\
\text { motorcycle model }\end{array}$ \\
\hline $\begin{array}{c}\text { calculation of } \\
\text { intermediate variables }\end{array}$ & multi-body simulation \\
\hline braking simulation & $\begin{array}{c}\text { time domain } \\
\text { multi-body simulation }\end{array}$ \\
\hline $\begin{array}{c}\text { calculation of scalar indexes } \\
\text { that describe the 4 objectives }\end{array}$ & external spreadsheet \\
\hline
\end{tabular}

The entire project has tested the behavior of 1904 different suspensions (17 MOGA generations). Each design took about 2.5 minutes, hence total CPU time was 80 hours.

\section{Optimization Results}

modeFRONTIER stores input variables, derived variables and output variables in a design table, whose rows reflect the tested configurations. Such a table is then processed to extract key information about the optimization scenario.

Main statistical relationships between variables (inputinput, input-output, and output-output) may be investigated by plotting the correlation matrix. The example proposed in Fig. 5 has been created to check for possible links between the 4 optimization objectives. The bottom-left section of the matrix shows the correlation factors, which provide a 
statistical measure of the linear association between objectives. Null, or small correlation indexes mean that there is no clear trend between the variables, whereas value +1 or close to +1 (or -1 ) indicate good direct (or inverse) correlation.

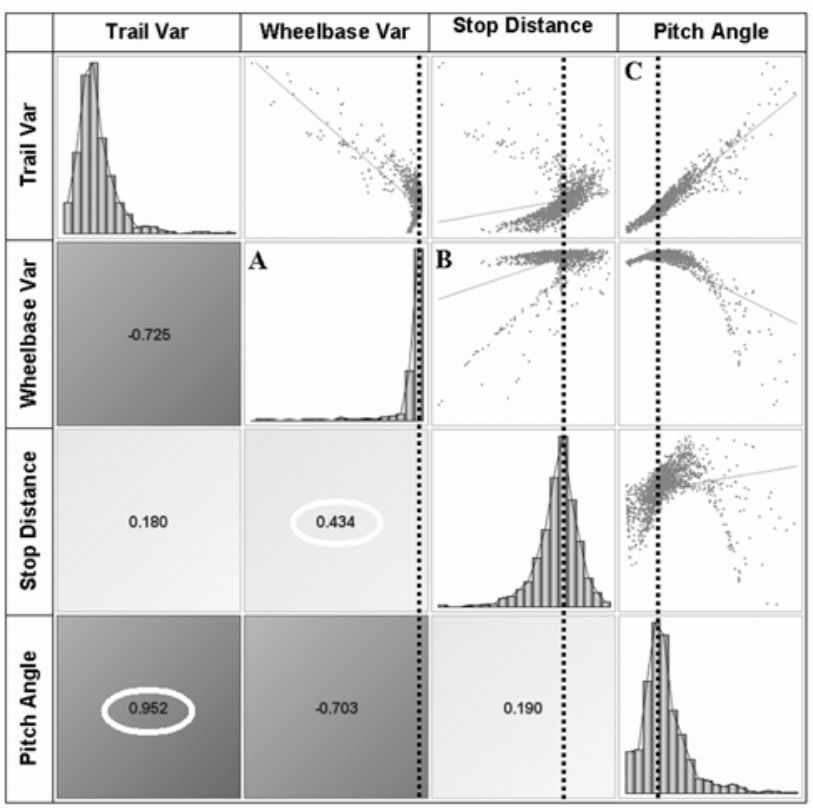

Fig. 5. Correlation matrix for the objectives

There are clear negative correlations between trail variation and wheelbase variation, as well as between pitch angle and wheelbase variation. Statistically, this means that when the wheelbase shortening is small, both trail shortening and pitch angle tend to be more pronounced.

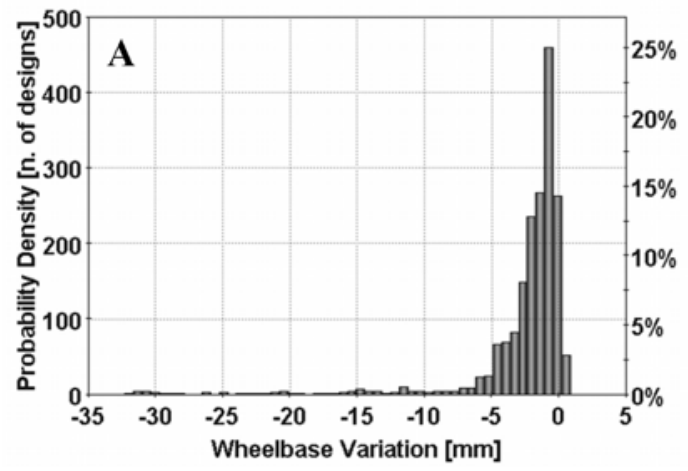

Fig. 6. Probability Density Function for the wheelbase reduction objective

Diagonal cells illustrate the Probability Density Function Chart for each objective. They allow the identification of trends that may not be obvious. For example, the wheelbase variation distribution is strongly asymmetric, with higher probability density on the right side of the total range, from $-65.0 \mathrm{~mm}$ to $+0.8 \mathrm{~mm}$. Since the optimizer was asked to limit the wheelbase shortening, the result is very satisfactory: more than $95 \%$ of motorcycles exhibit a wheelbase reduction between $-5.0 \mathrm{~mm}$ and $0.0 \mathrm{~mm}$ (Fig. 6). Other objec- tives are characterized by wider distributions, with less strong trends.
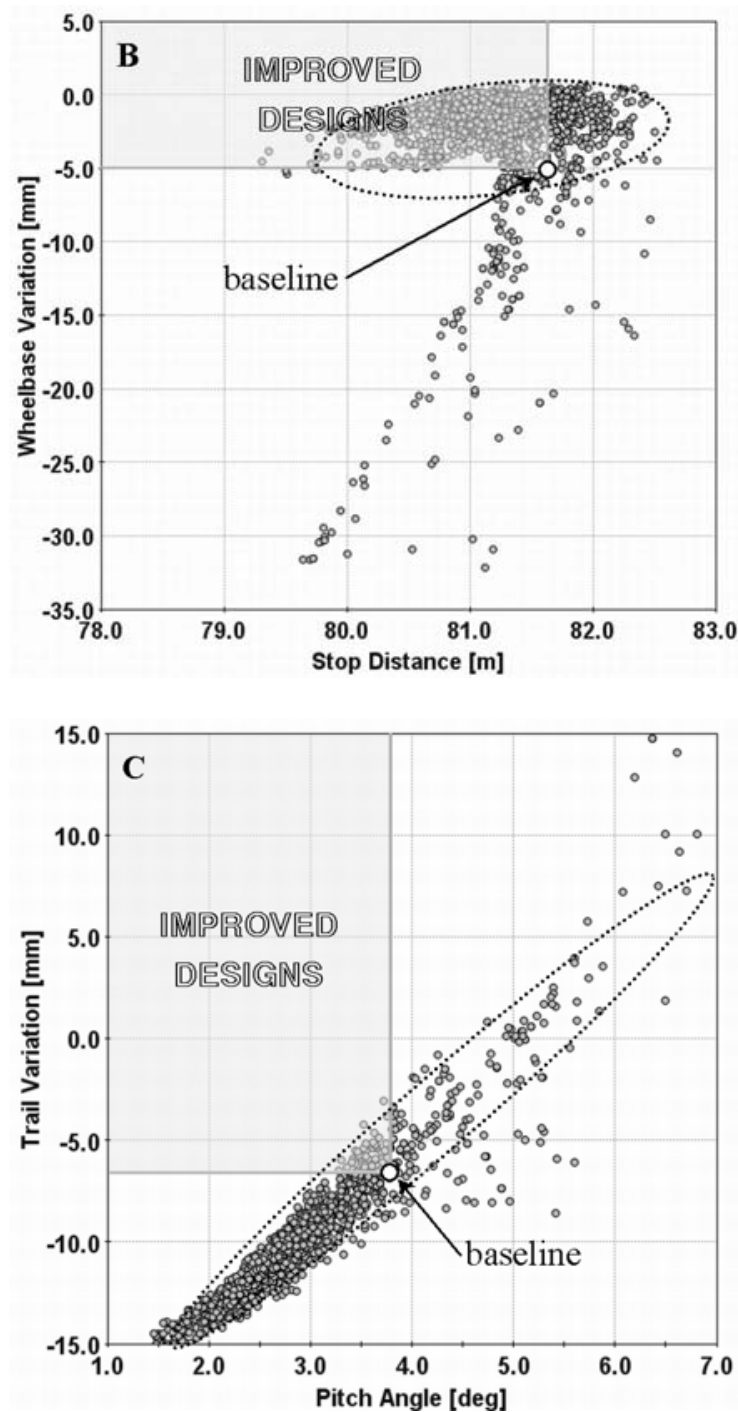

Fig. 7. Design evaluation

The upper-right section of the correlation matrix contains scatter charts, which represent the design distribution on 2D Cartesian graphs having $\mathrm{X} \& \mathrm{Y}$ axes in accordance with row \& column of the matrix. Two of these charts are highlighted and zoomed in Fig. 7, for a better understanding.

The upper plot shows the connection between stopping distance and wheelbase. Design spots lie in a wide cloud, thus confirming a low correlation level $(0.434)$. Trail variation and pitch angle, in contrast, are strongly correlated (0.952) as proved by the bottom plot, where design spots substantially lie on a narrow inclined area.

Besides examining object correlations, it is essential to analyze the relationships between objectives and input parameters. modeFRONTIER provides specific tools to rate the importance of each parameter, which is the first step towards a simplification of the problem.

As an example, Fig. 8 shows the t-Student chart for the stopping distance objective. The input parameters are listed from left to right, according to their effect on the chosen objective. The strongest (inverse) effect is given by the parameter which defines the position of the attaching point of 
the spring-damper assembly on the lower link of the suspension (see Fig. 1). In order to reduce the stopping distance, such point should be placed as forward as possible.

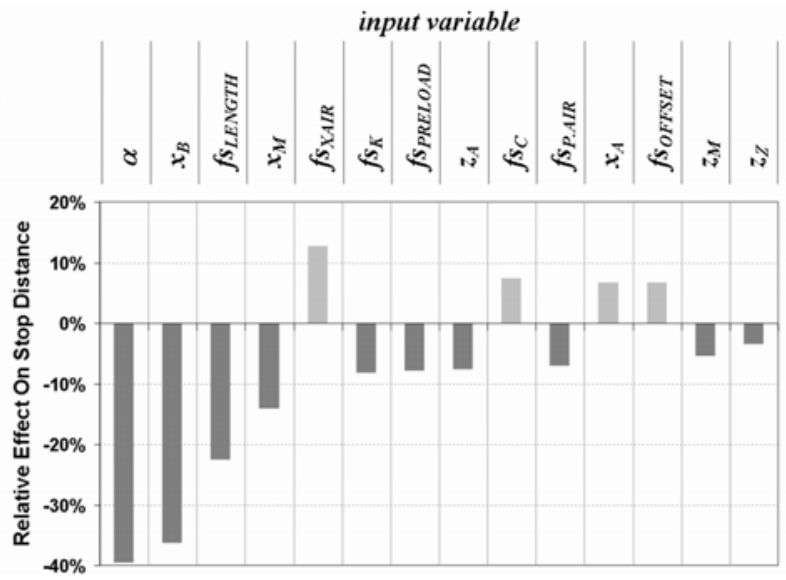

Fig. 8. Student chart: Effects of design parameters on stopping distance (see Fig. 1 and Fig. 2 for labels)

\section{Selecting the Best Design}

In order to understand how effective the optimization has been, it is necessary to compare the designs, focusing on objective values. Fig. 9 shows a parallel chart, where a graphical filter is used to hide all designs that do not meet one or more optimization goals. Considering simultaneously the 4 optimization criteria, the 13 visible designs of Fig. 9 are better than the baseline model. This selection is rather small ( $0.68 \%$ of total), meaning that the baseline motorcycle was already well designed for the objectives we have chosen.

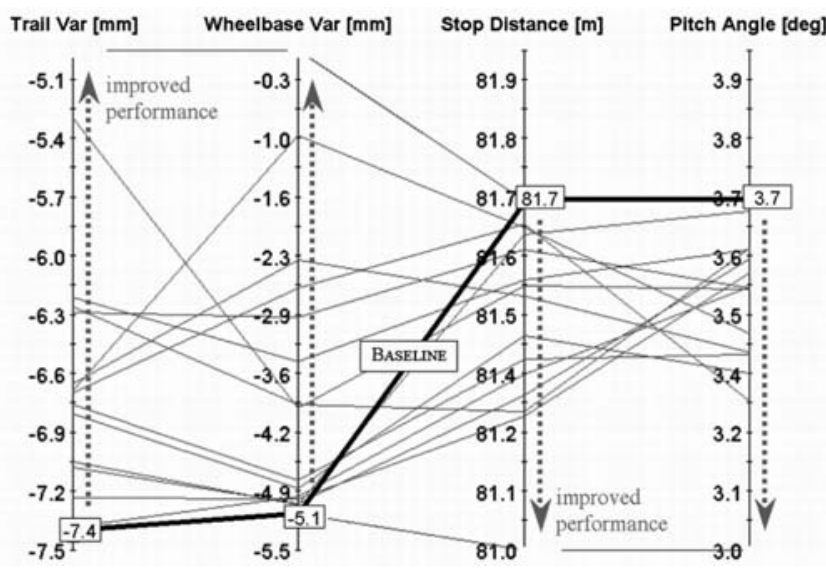

Fig. 9. Parallel chart to identify the best design

Theoretically the best design - among all - belongs to this sub-set. However, it can be observed that the only noticeable improvement concerns the wheelbase variation (from $5.1 \mathrm{~mm}$ to $-0.9 \mathrm{~mm}$ ). The gains for the other objectives, including the reduction of the stopping distance, are rather poor. To be fair, none of the 13 selected configurations improves the braking behavior sufficiently to justify a review of the baseline design.
Although at first glance it appears that the optimization failed, this may be a precipitate conclusion. In most cases, trade-off relationships exist between the objectives meaning that a slight loss in secondary ones opens a new scenario where the priority goal can be reached. modeFRONTIER includes a Multi Criteria Decision Making tool that uses pre-defined priorities to drive the choice in similar situations.

By applying such tool to the full design table, two "best" candidate designs have been selected; their performances are summarized in Table 3.

Table 3 Performances of best candidate designs

\begin{tabular}{llllll}
\hline QUANTITY & BASELINE & DES. 566 & \multicolumn{2}{l}{ DES. 792 } \\
\hline trail var & $-7.4 \mathrm{~mm}$ & -1.1 & $-85 \%$ & -15.2 & $+105 \%$ \\
wheelbase var & $-5.1 \mathrm{~mm}$ & -31.6 & $+519 \%$ & -3.8 & $-25 \%$ \\
stopping dist & $81.7 \mathrm{~m}$ & 79.6 & $-2.5 \%$ & 79.4 & $-2.8 \%$ \\
pitch angle var & $3.7 \mathrm{deg}$ & 5.2 & $+40 \%$ & 1.8 & $-51 \%$ \\
\hline
\end{tabular}
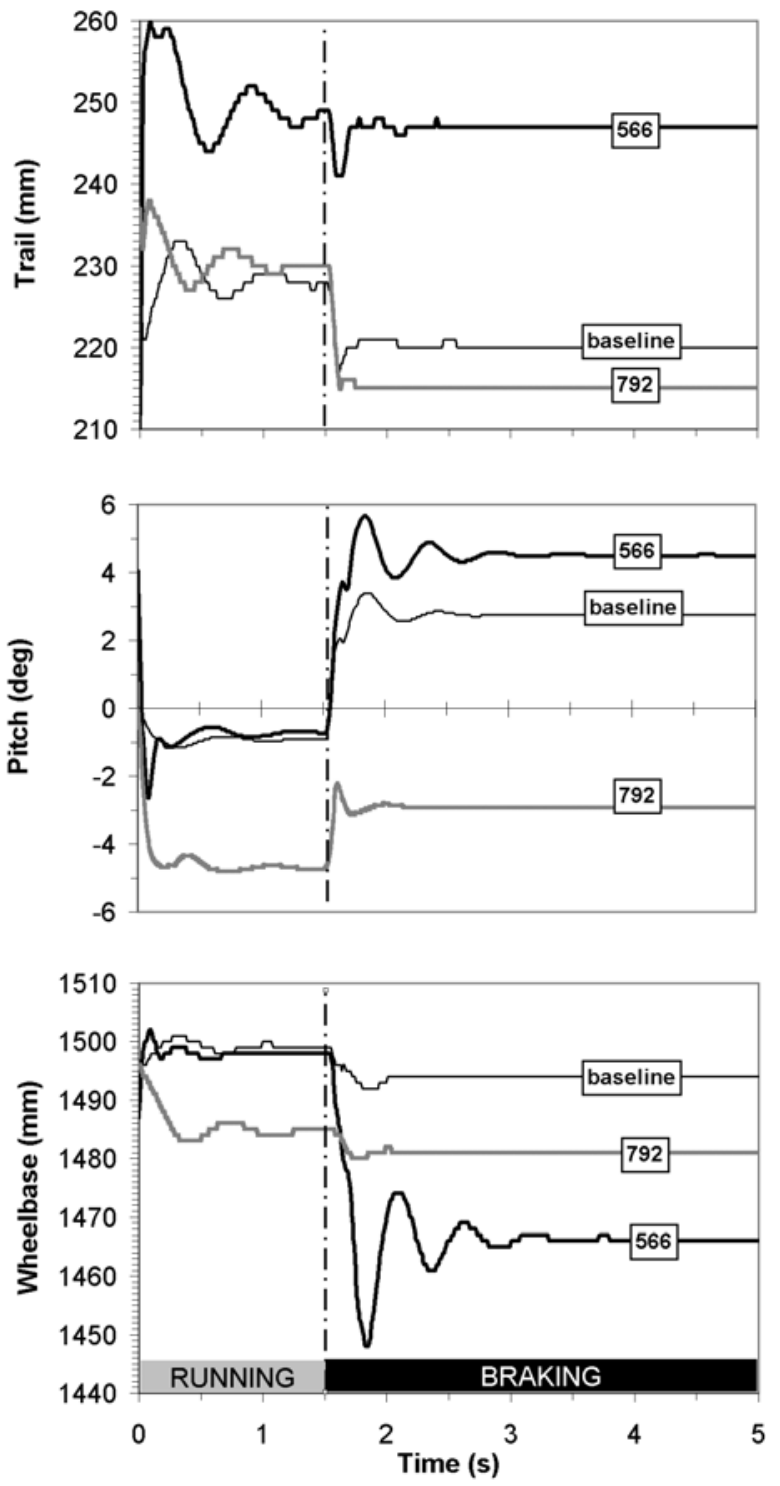

Fig. 10 Comparison between baseline and both optimized designs 
Both solutions provide a significant reduction of the stopping distance which is an important step forward in terms of safety. Such positive results can only be reached provided that, in braking condition, either the shortening of the wheelbase (des. 566) or the shortening of the mechanical trail (des. 792) gets more pronounced with respect to the corresponding shortening measured on the baseline motorcycle. The first selected configuration (des. 566) also causes a slight increase in pitching motion.

To make a definitive choice, it is useful to compare both optimized motorcycles with the baseline model.

The Fig. 10 clearly shows that design 566 does not differ too much from the starting vehicle, except for the trail that is initially longer. However, the suspension of this design is too soft and too low which causes undesired effects, such as front diving, wheelbase shortening, and lasting oscillations. Design 792, however, is quite different from the baseline motorcycle, e.g. the initial wheelbase is about $15 \mathrm{~mm}$ shorter and the chassis is inclined 4.7 deg forward. Suspension characteristics now appear adequate to prevent oscillations and excessive diving.

Finally and according to the optimization criteria, design 792 is the best choice. Presumably, the reduction of the stopping distance is not linked to the suspension update, but to the shorter wheelbase. Indeed, assuming that the ABS fixes the longitudinal slip, the braking force becomes proportional to the vertical load on the front tire, which is inversely proportional to the motorcycle length. To complete the research, both overall stability and handling capabilities should be investigated using a more sophisticated 3D model.

The design 792 saves about $2.3 \mathrm{~m}$ in stopping distance, which might be crucial in emergency maneuvers. Other objectives are less important, although improvements have been obtained as well, confirming the efficiency of the Multi-Objective optimizer.

Apart from the suggested application, this paper presents an innovative methodology for the design approach. modeFRONTIER provides capabilities for automatic and fast investigation of the various alternatives and offers smart tools to extract key information from the generated data.

\section{Conclusions}

This work has demonstrated the effectiveness of multiobjective optimization techniques in improving vehicle dynamics. The main parameters of a motorcycle front suspension have been tuned to shorten the braking distance, increasing, at the same time, the stability and the comfort. Despite these objectives are opposed each other, it has been possible to identify a good solution among the 1900 tested.

The quality of the optimization strongly relies on the multi-body model accuracy, which needs to capture (at least) the correct trends in the vehicle response. The second crucial step is the definition of suitable scalar indexes, which have to univocally measure the performances of the vehicle, starting from time-variable kinematical quantities.

The proposed methodology, which relies on modeFRONTIER capabilities, can be easily generalized and applied to any vehicle type, whose behavior is reproducible through parametric multi-body simulation.

\section{References}

1. V. Cossalter, R. Lot, F. Maggio, The influence of tire properties on the stability of a motorcycle in straight running and in curve, SAE Paper 001-014, 2002.

2. E.J.H. De Vries and H.B. Pacejka, The effect of tire modeling on the stability analysis of a motorcycle, In proc. $4^{\text {th }}$ International symposium on Advanced Vehicle Control (1998).

3. R. Lot, A motorcycle tire model for dynamic simulations: theoretical and experimental aspects, proc. $3^{\text {rd }}$ International Tribology Conference, (2002).

4. V. Cossalter R. Lot, F. Maggio, On the stability of motorcycle during braking, SAE Paper 2004-32-0018, 2004.

5. R.S. Sharp, The stability and control of motorcycles, Journal of Mechanical Engineering Science, 13, 316-329 (1971).

6. V. Cossalter, R. Lot, A motorcycle multi-body model for real time simulations based on the natural coordinates approach, Vehicle System Dynamics, 37, 423-448 (2002).

7. V. Cossalter, R. Lot, F. Maggio, A multibody code for motorcycle handling and stability analysis with validation and examples of application, SAE Paper, 2003-32-0035, 2003.

8. F. Biral, M. Da Lio, Modeling drivers with the optimal maneuver method, In proc. of $7^{\text {th }}$ ATA International Conference \& Exhibition, (2001).

9. F. Biral, M. Da Lio, F. Maggio, How gearbox ratios influence lap time and driving style - an analysis based on time-optimal maneuvers, SAE Paper 2003-32-0056, 2003.

10. D. Bortoluzzi, R. Lot, and N. Ruffo, Motorcycle steady turning: the significance of geometry and inertia, In proc. of $7^{\text {th }}$ ATA International Conference \& Exhibition, (2001).

11. M. Da Lio, V. Cossalter, D. Bortoluzzi, F. Biral, Experimental and theoretical study of motorcycle transfer functions for handling evaluation, Vehicle System Dynamics, 39 , 1-25 (2003).

12. R. Lot, A motorcycle tire model for dynamic simulations: theoretical and experimental aspects, $3^{\text {rd }}$ International Tribology Conference, (2002).

13. V. Cossalter, Motorcycle Dynamics, 2006, ISBN 978-14303-0861-4

14. D.J.N. Limebeer, R.S. Sharp, S. Evangelou, The stability of motorcycles under acceleration and braking, Journal of Mechanical Engineering Science, 215, 1095-1110 (2001).

15. V. Cossalter., R. Lot, F. Maggio, The modal analysis of a motorcycle in straight running and on a curve, Meccanica, 39, 1-16 (2004).

16. H.B. Pacejka, E. Bakker, The magic formula tire model, Vehicle System Dynamics 21, 1-18 (1992). 